Universidad de Lima

Facultad de Ciencias Empresariales y Económicas

Carrera de Economía

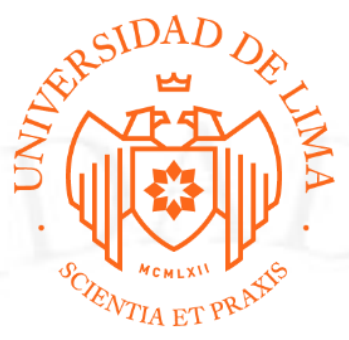

\title{
CASO DE ESTUDIO: CRISIS FINANCIERA INICIADA EN EL 2008
}

Trabajo de suficiencia profesional para optar el Título Profesional de Economista

Julio Andre Martinez Diaz

Código 20091677

\section{Asesor}

Dante Urbina Padilla

Lima - Perú

Agosto de 2019 
CASE STUDY: FINANCIAL CRISIS INITIATED IN 2008 


\section{TABLA DE CONTENIDO}

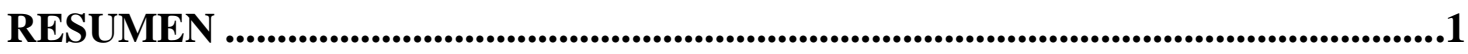

ABSTRACT

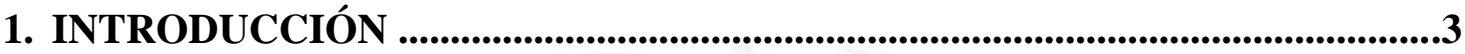

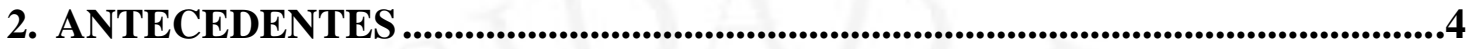

2.1 Prueba de Eficiencia de Fama................................................................

2.1 Prueba de Anomalías de mercado ......................................................................

2.1.1 Prueba de Efecto Fin de Semana....................................................................

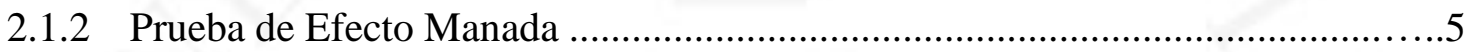

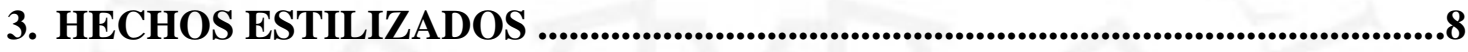

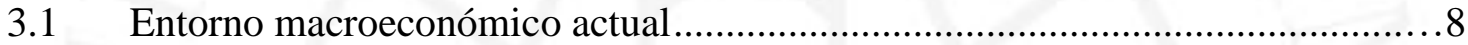

3.2 El desarrollo del Mercado bursátil peruano y colombiano ..............................10

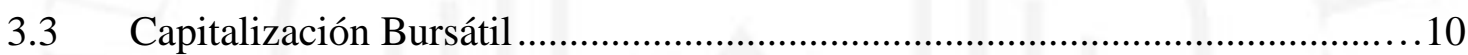

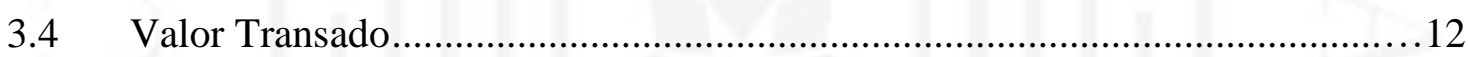

3.5 Participantes de los mercados de capitales (BVL y BVC) ..............................14

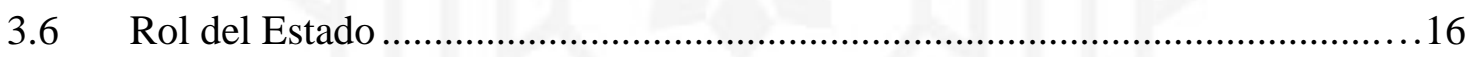

4. MARCO TEÓRICO...........................................................19

4.1 Hipótesis de Eficiencia de Mercado de Capitales ............................................19

4.2 Supuestos de la hipótesis de mercado eficiente ............................................21

4.3 Anomalías de mercado: Efecto fin de semana y Efecto manada ......................22

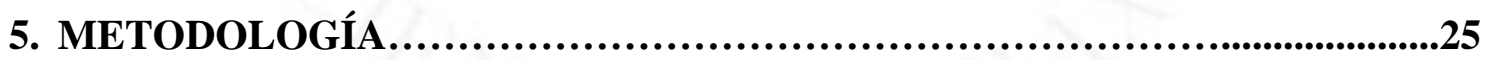

6. ANÁLISIS.....................................................................27

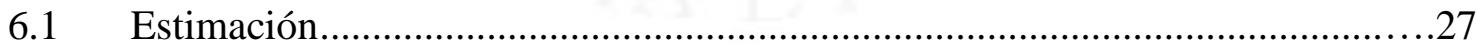

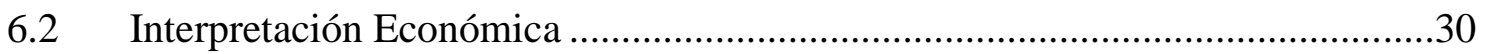

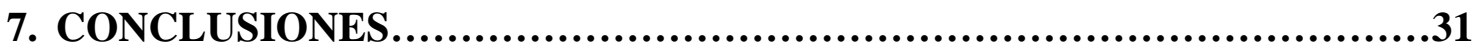

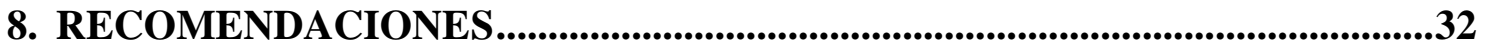

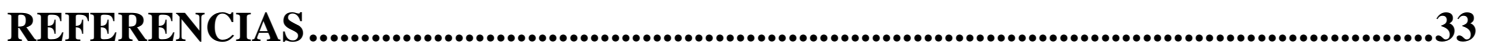

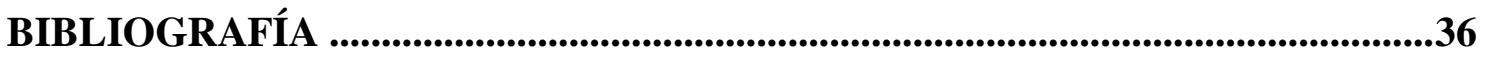




\section{ÍNDICE DE TABLAS}

Tabla 6.1 Mínimos Cuadrados a la CSSD del IGBVL25 con variables DL y DU, 20082018 .27

Tabla 6.2 Mínimos Cuadrados a la CSSD del COLCAP con variables DL y DU, 20082018 .27

Tabla 6.3 Test de Raíz Unitaria al IGBVL 25, 2008-2018 ….....................................28

Tabla 6.4 Test de Raíz Unitaria a COLCAP, 2008-2018 …........................................28

Tabla 6.5 Test de Varianza a IGBVL 25 ....................................................................29

Tabla 6.6 Test de Varianza a COLCAP ...........................................................................29 


\section{ÍNDICE DE FIGURAS}

Figura 3.1 Crecimiento económico real de algunas de las principales economías, 20178

Figura 3.2 Capitalización Bursátil en millones de dólares, 2008-2018 ......................... 11

Figura 3.3 Volumen Negociado de la BVL y BVC en millones de dólares, 2010-2016 13

Figura 3.4 Fondo Administrado como porcentaje del PBI, 2012 …............................ 14

Figura 3.5 Participación de Inversionistas cómo \% del PBI, 2016............................... 15

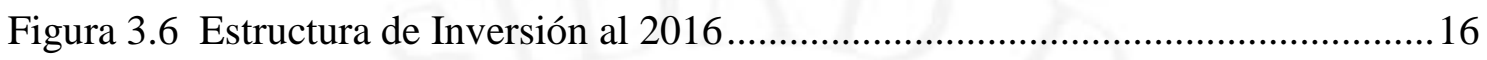




\section{ÍNDICE DE ANEXOS}

Anexo 1: Matriz de Operacionalización para Efecto Manada ......................................... 38 


\section{RESUMEN}

La presente investigación se centra en analizar una anomalía que ocurre en los mercados bursátiles conocida como el Efecto Manada (Hott, 2009), este efecto se define como la posibilidad de que las decisiones tomadas por un grupo de inversionistas tengan un efecto de contagio en otro grupo y que de esta manera ellos se guíen por estas decisiones para invertir dejando de lado el análisis fundamental y contradiciendo el supuesto de racionalidad de (Fama, 1970). Ambos casos tomaron relevancia a partir de la crisis iniciada en el 2008 y son materia de estudio en la nueva corriente de economía conductual.

De esta manera, se evidenciará el porqué es necesario aumentar los estudios en las anomalías de los mercados de capitales, principalmente los de Perú y Colombia para entender cómo estas afectan las condiciones que estos mercados tienen y que además se evidencian principalmente durante las crisis financieras.

Los primeros 4 capítulos contienen las generalidades de la investigación. Comenzando con el primero, que explica la relevancia de la investigación y el impacto de la conducta de los inversionistas ante situaciones de crisis; a continuación, en el segundo capítulo se presentan antecedentes. Luego, los capítulos 3 y 4 muestran hechos estilizados en ambos países y el marco teórico. Finalmente, los capítulos del 5 al 8 muestran la metodología, análisis, conclusiones y recomendaciones.

Para ambos mercados de capitales, se halla la evidencia de que sí existe un efecto manada post crisis financiera iniciada en el 2008.

Palabras clave: crisis financiera mundial, anomalías de mercados de capitales, efecto manada, finanzas conductuales. 


\begin{abstract}
The present investigation focuses on analyzing an anomaly that occurs in the stock markets known as the Herding Effect (Hott, 2009), this effect is defined as the possibility that the decisions taken by one group of investors have a contagion effect on another group and that in this way they are guided by these decisions to invest leaving aside the fundamental analysis and contradicting the rationality assumption of (Fama, 1970). Both cases took relevance from the crisis that began in 2008 and are a matter of study in the new current of behavioral economics.

In this way, it will be evident why it is necessary to increase studies on anomalies of capital markets, mainly those of Peru and Colombia to understand how these affects the conditions that these markets have and which are mainly evidenced during financial crises.

The first 4 chapters contain the generalities of the investigation. Starting with the first, which explains the relevance of the investigation and the impact of investors' behavior in crisis situations; Then, the second chapter presents background information. Then, chapters 3 and 4 show stylized facts in both countries and the theoretical framework. Finally, chapters 5 through 8 show the methodology, analysis, conclusions and recommendations.
\end{abstract}

For both capital markets, there is evidence that there is a herd effect after the financial crisis that began at 2008 .

Keywords: global financial crisis, capital markets anomalies, herd effect, behavioral finance. 


\section{INTRODUCCIÓN}

El objetivo de esta investigación es verificar si luego de iniciada la crisis financiera en el 2008, se cumple la anomalía del mercado bursátil conocida como efecto manada (Shiller, 2003), a través de un estudio para el caso peruano y colombiano, tomando como base el Índice General de la Bolsa de Valores de Lima 25 (IGBVL25) y el Índice Bursátil de Colombia (COLCAP), estos abarcan las cotizaciones de las empresas más rentables de la bolsa de valores entre los años 2008-2018. Esto servirá también para resaltar la importancia del peso especulativo que conllevó a que algunas empresas presenten rendimientos anormales durante la crisis del 2008.

Para esto, se ha desagregado este objetivo general en tres objetivos específicos, a fin de detallar cual es el fin del presente trabajo, los cuales son:

- Describir la teoría de la Hipótesis de Eficiencia de Mercado de Capitales y los estudios de finanzas conductuales respecto a las anomalías de mercado que se presentan en esta teoría.

- Explicar el contexto macroeconómico en el que se encuentran los mercados bursátiles de Perú y Colombia.

- Verificar la existencia del comportamiento manada en el mercado bursátil peruano y colombiano en el periodo post crisis financiera a fin de reconocer el comportamiento de los agentes económicos en esa época.

Así mismo, la hipótesis que surge a partir de toda la literatura revisada es que la contrastación de los diferentes modelos permite apreciar que se cumple la propuesta teórica del efecto manda.

Del mismo modo que con los objetivos, se han trazado una hipótesis específica a ser tratada, la cual es:

- En la Bolsa de Valores de Lima (BVL) y en la Bolsa de Valores de Colombia (BVC) se cumple la teoría respecto al efecto manada que ocurre en los mercados de capitales (sobre todo emergentes) y que es considerada como una anomalía presente en estos mercados. 


\section{ANTECEDENTES}

\subsection{Prueba de Eficiencia de Fama}

La prueba de eficiencia en su forma débil afirma que no existe una relación entre los rendimientos pasados y futuros de los activos. Al respecto, numerosos son los estudios que han evaluado las condiciones de eficiencia de Fama (1970), desde estudios para los mercados desarrollados (Estados Unidos y Europa) como también para los emergentes (Asia, África, Latinoamérica y Oceanía). Sin embargo, el que más veces ha sido estudiado por su tamaño e importancia ha sido la bolsa norteamericana. A continuación, se presentan los trabajos más importantes por región:

En Norteamérica, Shynkevich (2012), combina técnicas y utiliza el análisis técnico y el tamaño de la empresa para evaluar los índices NYSE, AMEX y NASDAQ, concluyendo que, los precios de las acciones de las empresas pequeñas y grandes, a partir del año 2000 comienzan a ser más difíciles de predecir, siendo esto un indicador de la mejora de la eficiencia.

En Europa, esta hipótesis ha sido contrastada mediante diversos métodos, al igual que en Estados Unidos. Entre los más recientes, destacan los trabajos de Straßburg, Gonzáles y Alexandrov (2012), quienes fueron capaces, mediante técnicas de valoración con algoritmos, de predecir los precios futuros y obtener rendimientos extraordinarios entre 1986 y 2004.

Al mismo tiempo, Khan y Vieito (2012), analizan los mercados de las bolsas de Lisboa-Porto y Euronext, y concluyen, mediante pruebas de paseo aleatorio, que la bolsa ha mejorado su eficiencia luego de la fusión de ambas en el año 2002.

En Latinoamérica, Mendez (2009), analiza el mercado bursátil de Colombia para determinar si la bolsa de ese país fue eficiente en su forma débil mediante pruebas de camino aleatorio y varianza, concluyendo que el mercado bursátil colombiano no ha sido eficiente ya que la serie no cumple con el supuesto de la caminata aleatoria.

Por otro lado, Morales, Ruíz, y Gutiérrez (2015), utiliza otra metodología denominada estudio de eventos, para analizar cómo se ajustaban los precios a la información pública que se emite de manera anual, para así contrastar el método con el 
supuesto de la forma semi-fuerte de eficiencia, y concluyendo que el mercado accionario colombiano no ha sido eficiente en el periodo estudiado.

\subsection{Prueba de Anomalías de mercado}

Dentro de las anomalías, se han encontrado los siguientes estudios que han sido desarrollados por autores alrededor de todo el mundo:

\subsubsection{Prueba de Efecto Fin de Semana}

Respecto a esta anomalía en la literatura encontramos que en Sudamérica Rodríguez (2012), analizó los índices de las principales Bolsas de Sudamérica entre los cuales están Brasil, Chile, Colombia, México, Argentina y Perú para buscar la presencia del efecto día de semana y concluyó que en algunos de estos mercados se evidencia que dependiendo del día de semana que se invierta se pueden obtener mejores rendimientos. También resaltó que esta anomalía aparece principalmente en las Bolsas de los mercados emergentes.

\subsubsection{Prueba de Efecto Manada}

Esta anomalía de mercado ha sido un tema de estudio abarcado por muchos investigadores y entre ellos existen varios pertenecientes al área de finanzas y afines.

Puesto que este comportamiento puede afectar los niveles de riesgo y retorno, el efecto Manada ha sido modelado, tomando como variable de estudio la dispersión y ha sido evaluado estudiando el mercado en general.

En los inicios del año 2000, Chang, Cheng y Khorana (2000), analizaron la desviación absoluta de sección cruzada (Cross Sectional Standard Deviation o CSSD) como medida de dispersión de los rendimientos del mercado bursátil de Estados Unidos, Hong Kong, Corea del Sur y Taiwán entre los años 1963-1997 concluyendo que existe un efecto manda en todos menos en Estados Unidos.

A partir de este modelo, múltiples investigadores han estudiado este efecto en distintos mercados bursátiles de todo el mundo. Al respecto, Chen (2013), tomó como muestra los índices de 69 países entre los años 2000-2009 y quien además los clasificó 
en grupos por países desarrollados, emergentes y de frontera, concluyendo que existe el efecto manada en la mayoría de países, siendo más propenso su aparición cuando la perspectiva económica está a la baja.

Por otro lado, en el mismo año, Gebka y Wohar (2013), concluyeron que se afirmaba la presencia del efecto manada, tras analizar a 9 sectores de 32 países entren los años 1998-2012, en distintos sectores como el de materias primas y el de servicios.

En Europa, se encuentra el trabajo de Henker, Henker y Mitsios (2006), quienes buscaron la presencia de esta anomalía analizando a las 160 compañías de mayor actividad y de capitalización bursátil del Índice ASX2000 de Australia, concluyendo que se rechazaba la hipótesis de la existencia de este efecto lo cual significa que la información se difunde de manera eficiente lo cual se refleja en la toma de decisiones de los inversionistas de esta Bolsa.

Más adelante, Ouarda, Bouri y Bernard (2013), usaron como muestra a las 174 empresas (distribuidas en 10 industrias) de mayor capitalización bursátil del EuroStoxx600, tomando como muestra a las cotizaciones mensuales entre los periodos 1998-2010 y concluyendo que este efecto se podía apreciar en todas las industrias de la bolsa europea, menos en la industria de bienes de consumo, siendo más evidente su presencia en la época de la crisis mundial del 2008.

En paralelo a estas investigaciones, en Asia, Lao y Singh (2011), investigaron la presencia de este efecto en el Shanghái A-share Índex (conformado por las acciones que están bajo el poder de inversionistas locales) y en el Bombay Stock Exchange Índex, entre los años 1999-2009, son los índices que mejor representan las bolsas de China e India respectivamente, concluyendo que en ambos se evidenciaba la presencia de este efecto y que era más visible en ciertas circunstancias.

Más adelante, Liu (2013), utilizó cotizaciones diarias para evaluar la bolsa de Singapur, tomando como referencia a las acciones de mayor negociación entre los años 2002-2012, concluyendo que existe un efecto manada en esta bolsa y que se puede apreciar mejor este efecto durante la época de la crisis financiera.

Entrando en Latinoamérica, De Almeida, Costa y Da Costa (2012), utilizaron los principales índices de la región entre los años 2000-2010 tomando como referencia a las acciones de alto volumen de negociación, concluyendo que no se evidenciaba el efecto 
manada en la región. Sin embargo, en el caso específico de Chile, sí se detectó este efecto en los activos de alta y baja volatilidad. 


\section{HECHOS ESTILIZADOS}

\subsection{Entorno macroeconómico actual}

En el 2018, diez años después de iniciada la crisis financiera del 2008, como señala el reporte de estabilidad financiera del Banco de la República Bogotá (2018, págs. 13-17), "las principales economías mundiales han obtenido resultados positivos, excepto el Reino Unido”. Una de las más grandes, Estados Unidos, mostró ritmos de crecimiento más grandes que los previstos por la tasa de la política monetaria de ese país. Sin embargo, estos resultados no generaron un aumento significativo en los salarios y más bien generaron un aumento en las expectativas de inflación como se puede apreciar en el siguiente cuadro.

Figura 3.1

Crecimiento económico real de algunas de las principales economías, 2017

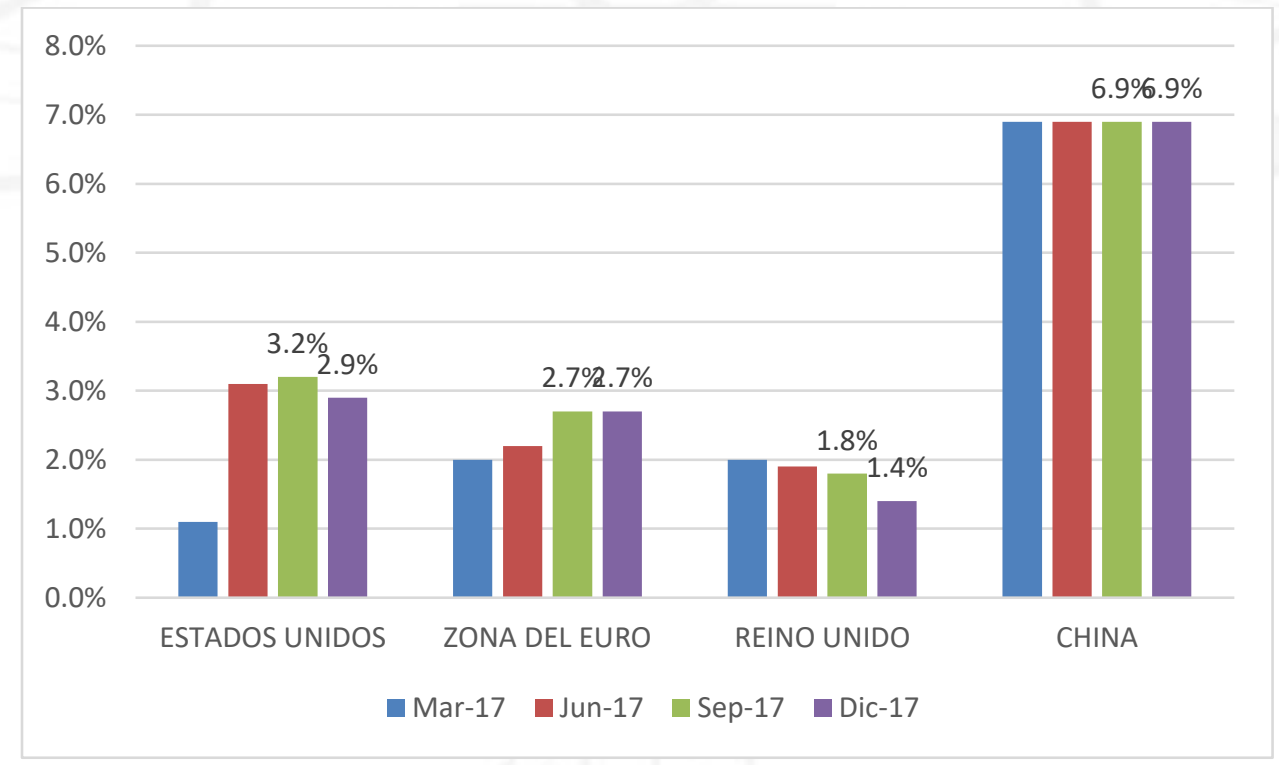

Fuente: Bloomberg (2019)

Elaboración propia

Además, las expectativas implícitas de la tasa de la política monetaria de Estados Unidos, puesto que se espera que las tasas de los fondos federales crezcan por encima del 2\% a partir del 2019 y esto levanto alertas sobre el crecimiento a mediano plazo, impactando negativamente el precio de las acciones de ese país. 
En la eurozona, se puede apreciar un crecimiento real de $2.7 \%$ a diciembre 2017 y dado que los niveles de inflación se mostraron estables, la tasa de política monetaria no se ha visto incrementada.

En el Reino Unido, todavía es preocupante los efectos negativos que pueda traer consigo la separación de este con la eurozona en términos de comercio internacional.

El país que más se expandió (China) si bien registra un crecimiento de $6.9 \%$ a finales del 2017, está pasando por un momento de muchas regulaciones relacionadas con la intermediación no bancaria.

Por otro lado, en américa latina se puede observar una tendencia similar, aunque países como Perú y México mostraron tasas más bajas a la del resto de las economías, explicado por choques exógenos como el fenómeno del niño costero del 2017 en el Perú y el sismo registrado en el mismo año para el caso de México. Lo anterior, sumado a los casos de corrupción e incertidumbre política, han generado alarmas y posibles pronósticos a la baja de dichas tasas.

Para el caso de Perú, se cerró con un crecimiento económico real de $2.2 \%$ en el 2017 y a partir del 2018 se espera tasas de crecimiento por encima del 4\% como menciona el reporte de estabilidad financiera del BCRP (Banco Central de Reserva del Perú, 2018).

Lo anterior podría modificarse si la guerra comercial entre Estados Unidos y China llega a intensificarse puesto que esto afectaría directamente la demanda de los productos como señala el reporte.

En el caso de Colombia, el reporte de estabilidad financiera del Banco de la República Bogotá (2018) señala que a pesar del bajo crecimiento que experimentaron el año 2017, se tiene una expectativa positiva en los términos de intercambio. 


\subsection{El desarrollo del Mercado bursátil peruano y colombiano}

Desde el año 2008, se puede apreciar que tanto como la BVL y la BVC han tenido una leve mejora respecto a épocas pasadas y que efectivamente existe una mayor dinámica que en tiempos previos a la crisis mundial del 2008.

No obstante, a nivel mundial, ambas todavía son consideradas como un mercado pequeño e incluso mirando a otras bolsas de la misma región, se puede apreciar que todavía se encuentran lejos de alcanzar a la capitalización bursátil de bolsas como la de Chile.

Por otro lado, en el reporte de estabilidad financiera del BCRP (2016), se observaba que el tamaño del mercado bursátil todavía es bajo lo cual evidencia que este mercado tiene poca profundidad y que además se ha ido desacelerando.

Ya en el 2018, se observa en el reporte de ese año del BCRP (2018), que, comparado con Colombia, las empresas en Perú tienen una mayor estabilidad financiera, lo cual se traduce en liquidez y rentabilidad; sin embargo, si bien en comparación con Colombia, Perú muestra mayor estabilidad, existe un bajo grado de profundización del mercado de capitales doméstico y con esto se evidencia la necesidad de aumentar el grado de liquidez y además del desarrollo de inversionistas institucionales.

\subsection{Capitalización Bursátil}

Luego de la crisis iniciada en el 2008, en el 2010, la capitalización bursátil de la BVLque mide el valor de las empresas listadas en el mercado bursátil entre acciones de capital e inversión- alcanzó aproximadamente un máximo histórico de 166 mil millones de dólares. Sin embargo, desde el siguiente año, la capitalización de la bolsa se ha venido desacelerando, esto debido a la menor participación de las empresas mineras.

Además, las empresas han utilizado este mecanismo de financiamiento (mediante la emisión de nuevas acciones) con menor regularidad como se puede apreciar en la frecuencia de negociación de las acciones listadas en la BVL. 
Similar ocurre en el caso de Colombia, en donde luego de haber experimentado una atracción de capital hasta el periodo del 2012, su capitalización bursátil ha ido decreciendo, esto a pesar de haber experimentado una recuperación desde el 2015.

Figura 3.2

Capitalización Bursátil en millones de dólares, 2008-2018

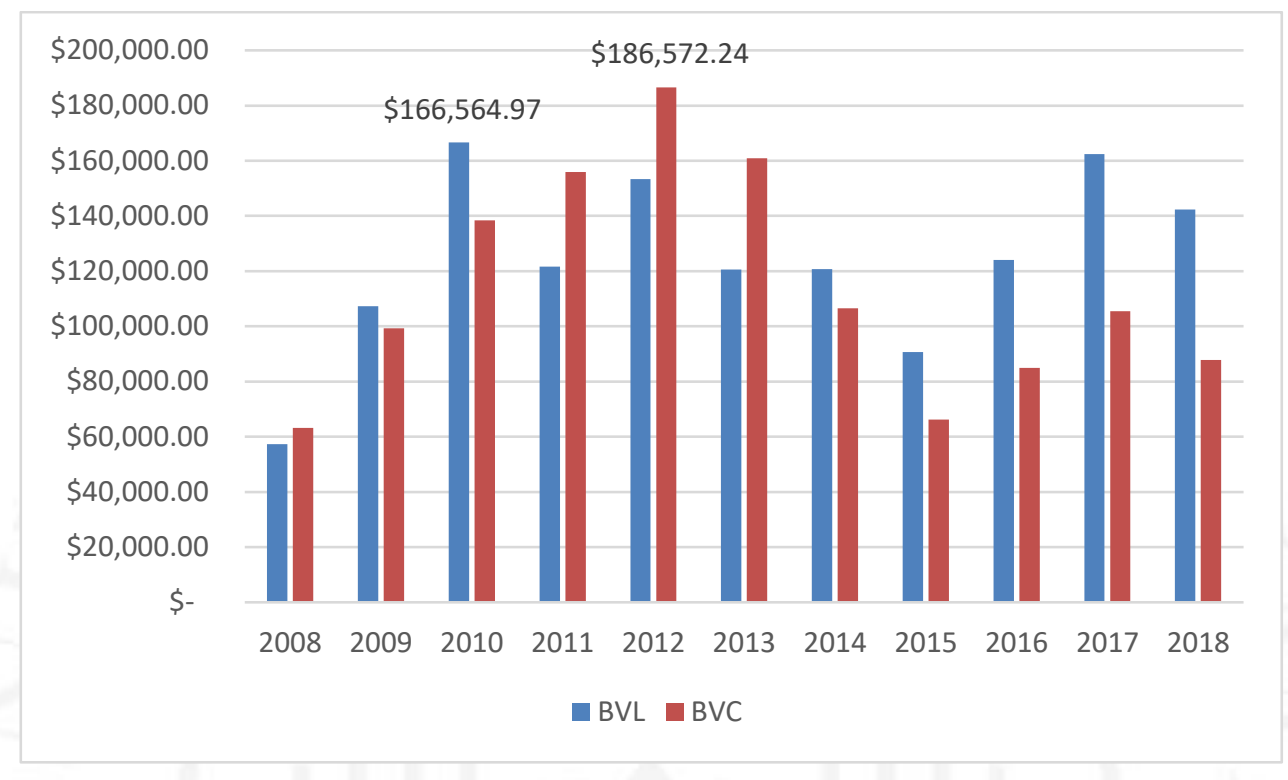

Fuente: Bloomberg (2019)

Elaboración propia

En las acciones de capital, lo más resaltante es el considerable incremento de la participación de los bancos y entidades financieras, que en los últimos 5 años han experimentado una subida de su capitalización de 6,305 millones a más 26,730 millones de dólares hacia finales del 2016.

Por otro lado, la participación de las compañías mineras se redujo de los casi 70,000 millones que alcanzó en el 2012 (lo cual representó en ese año un poco menos del $50 \%$ de la capitalización total de la BVL) a 34,895 millones de dólares en el 2015, lo cual representó una caída 50\% en 3 años.

Además, en el 2016 las acciones de inversión correspondientes a las compañías de este mismo rubro, cerraron en 808 millones de dólares, cifra mucho menor a los casi 2,700 alcanzados en el 2011. Esta considerable reducción de la capitalización de las mineras se debe probablemente a la caída de la proyección de este sector, ya que muchos proyectos se han paralizado y con lo cual los inversionistas han reducido su interés por estas acciones. 
Además, el informe estadístico de la BVL (2018), muestra que actualmente, en el mercado bursátil peruano existen 235 compañías con acciones y renta fija, 8 fondos de inversión y 40 empresas con solo renta fija, lo cual muestra que en los últimos años todo ha permanecido relativamente estable.

Sin embargo, cabe señalar que a pesar de que la capitalización de la Bolsa ha aumentado ligeramente respecto al 2015 , esta probablemente se dé más por el aumento de las empresas que cotizan respecto al de hace algunos años que por el incremento de la capitalización individual de las compañías que ya estaban registradas.

En resumen, el tamaño del mercado peruano, el cual es relativamente bajo, ha sido una limitación a la eficiencia de este, ya que en el año en el que le fue mejor a la BVL (2012, sin contar el histórico del 2010) su capitalización bursátil solo alcanzó el 48.56\% como muestra Sánchez (2013), en un reporte del BCRP.

\subsection{Valor Transado}

Continuando con el análisis de los principales indicadores de la BVL, se puede apreciar, en la gráfica elaborada con los datos recuperados de su página web, BVL (2016), que los volúmenes negociados se han reducido considerablemente. El total negociado, representado por la renta variable, se redujo desde los 6,000 millones de dólares que alcanzó en el 2010 a ser 2,700 millones hacia finales del 2016, como se mencionó en el capítulo, la reducción de las inversiones del sector minero fue una de las principales causas de la desaceleración de este sector. 
Figura 3.3

Volumen Negociado de la BVL y BVC en millones de dólares, 2010-2016

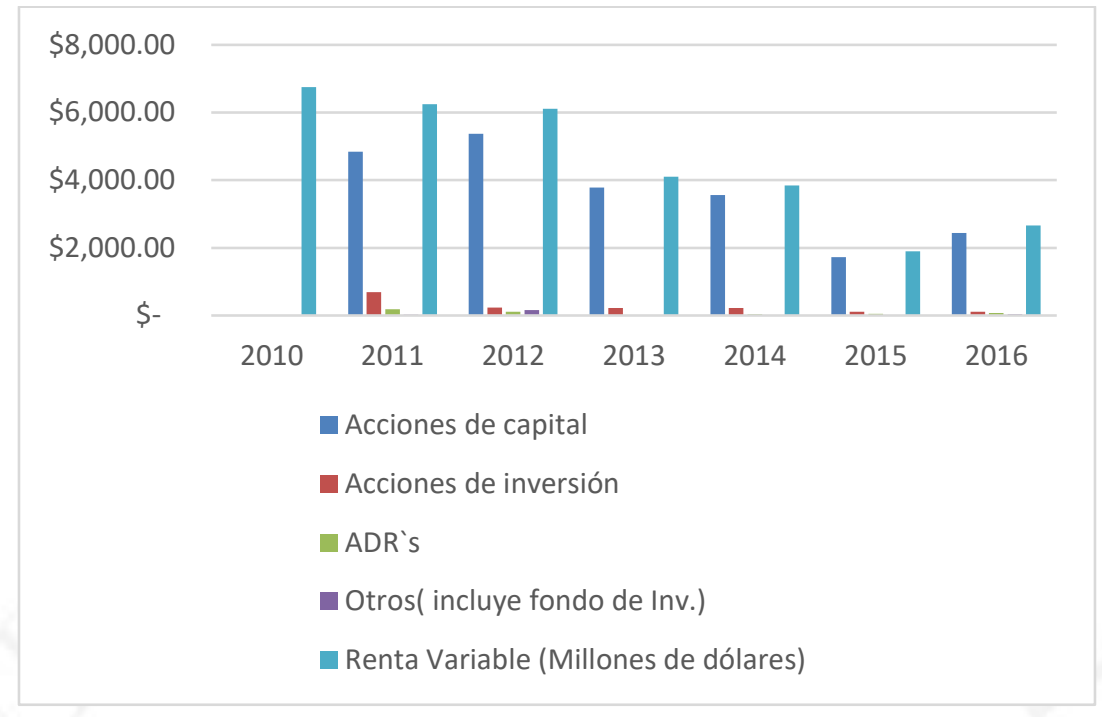

Fuente: BVL (2016)

Elaboración propia

Este gráfico ayuda a entender el des aceleramiento del crecimiento y eficiencia de la Bolsa ya que el volumen negociado es clave para que se cumpla la teoría de eficiencia de mercados bursátiles puesto que en un mercado eficiente las acciones son líquidas.

En cuanto a esto, se puede apreciar en el reporte de estabilidad financiera del BCRP (2016), que el grado de liquidez de este mercado sigue siendo bajo a nivel regional y mundial.

Además, el reporte señala que solo 8 de las 100 empresas más líquidas del mercado vieron como sus acciones fueron negociadas en más del 95\% de los días, debido en gran parte a que las empresas mineras fueron afectadas por la caída del precio de los productos entre los años 2011 y 2015 y también porque en el 2010 entro en vigencia la ley del impuesto a las ganancias de capital.

No obstante, a partir de enero de 2016, se exoneró este impuesto (de manera temporal) a las ganancias de capital, lo cual, sumado a la recuperación del precio de los productos a partir de ese año, impactó positivamente en los inversionistas ya que los volúmenes observados desde entonces comenzaron a incrementarse. 
Entonces, se puede ver como el poco número de participantes frecuentes del mercado bursátil es una limitación de la eficiencia de este mismo y también, otro factor el cual afectó a este mercado y a las negociaciones, fue el impuesto a las ganancias de capital.

En el caso colombiano, analizando la data en Bloomberg, se puede ver que el volumen negociado en Colombia ha pasado de 1,651 millones de dólares en el 2008 a incrementarse significativamente en los años siguientes. Si bien la BVC ha experimentado una contracción en los últimos años, se puede apreciar una mejora que hace que se mantenga alrededor de los 1,800 millones de dólares en el 2018.

\subsection{Participantes de los mercados de capitales (BVL y BVC)}

Dentro de los principales participantes de este mercado, encontramos a las Administradoras de Fondos de Pensiones, los Fondos mutuos y a las Compañías de Seguro.

Figura 3.4

Fondo Administrado como porcentaje del PBI, 2012

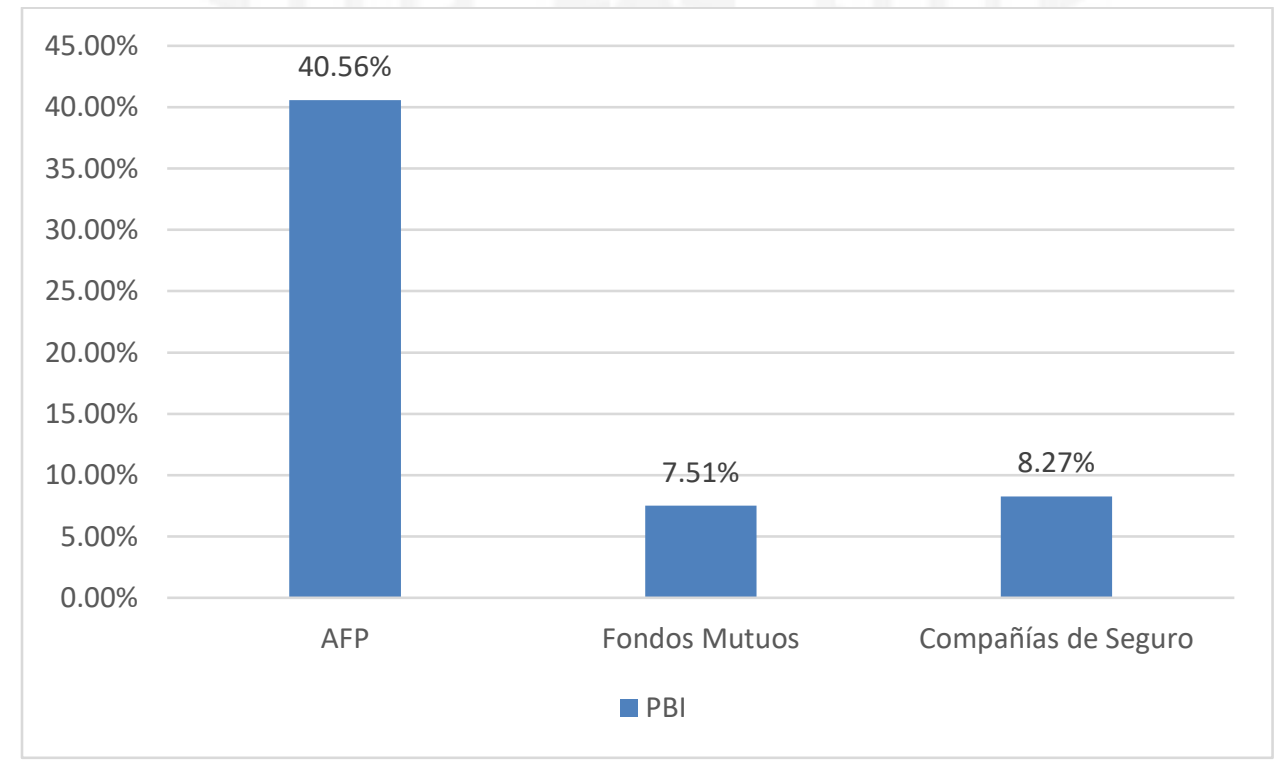

Fuente: BCRP (2013)

Elaboración propia 
- AFP. - Tan solo hasta el 2012, como señala Sánchez (2013), el fondo administrado por las AFP, que son las instituciones financieras que se encargan de administrar los fondos y ahorros de pensiones alcanzó el $40.56 \%$ del PBI.

- Fondos Mutuos. - En el mismo periodo, estos fondos (divididos en fondos 1,2 y 3) ocuparon tan solo el $7.51 \%$ del Producto Bruto Interno. Además, estas inversiones junto a las que realizan las Compañías de Seguros tienen menor monto invertido debido al mayor riesgo que estas inversiones traen.

- Compañías de Seguro. - Por su parte, estas últimas tienen un 8.27\% de capital invertido como porcentaje del PBI.

Figura 3.5

Participación de Inversionistas cómo \% del PBI, 2016

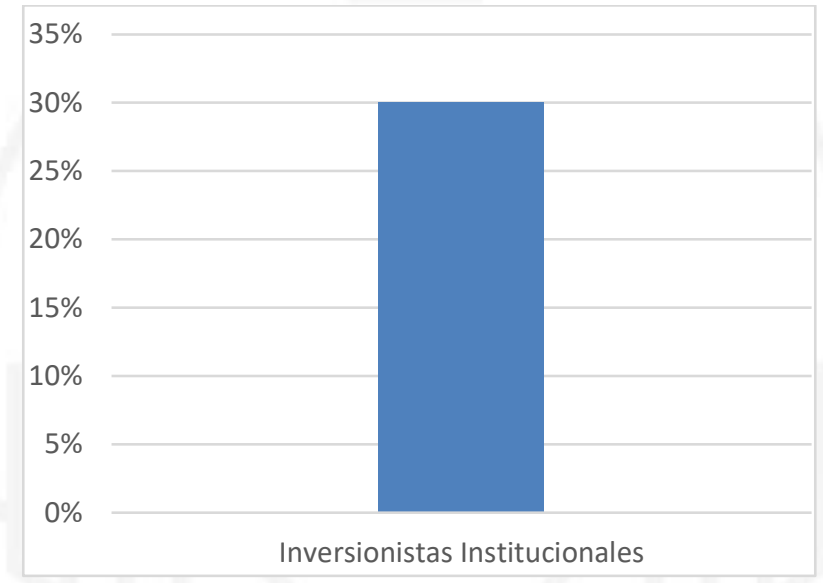

Fuente: BCRP (2017)

Elaboración propia

En comparación al año 2012 en específico (en el cual la BVL obtuvo su punto más alto cuando alcanzó una Capitalización Bursátil de 153,000 millones), hacia el 2016 el mercado de capitales peruano ha ido disminuyendo con lo cual, la participación de estas compañías se ha reducido considerablemente al punto de que entre estos tres grupos solo alcanza el 30\% del PBI como se muestra en el Reporte de Estabilidad Financiera del BCRP (2016) y manteniendo la tendencia del predominio de las AFP las cuales alcanzan el $70 \%$ del total de las inversiones institucionales, dejando a los Fondos mutuos con el $12 \%$ y a las Compañías de Seguro con $18 \%$. 
Figura 3.6

Estructura de Inversión al 2016

Estructura de Inversión al 2016

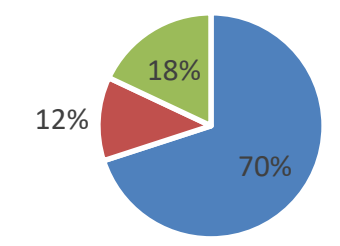

- AFP - Fondos Mutuos - Compañías de Seguro

Fuente: Superintendencia de Banca, Seguros y AFP, SBS (2017)

Elaboración propia

Sin embargo, existe una leve mejora respecto al 2015 probablemente por el incentivo hacia los inversionistas que generó la exoneración a las ganancias de capital a inicios del 2016.

Similar ocurre en el caso de Colombia, en donde también encontramos fondos de inversión colectiva que son el equivalente de las AFP, fondos mutuos y también las compañías de seguros.

\subsection{Rol del Estado}

Dentro de los organismos del Estado encargados de supervisar y regular el mercado de capitales peruano encontramos a 3 instituciones, las cuales son:

- Superintendencia del Mercado de Valores (SMV). - La cual se encarga de proteger a los inversores, ofreciendo transparencia y eficiencia de los mercados que regula.

- Superintendencia de Banca, Seguros y AFP (SBS). - Se encarga de supervisar de manera constante el Sistema financiero y además brinda las normas generales que las entidades deben de cumplir. 
- Banco Central de Reserva del Perú. - Su rol principal es el de controlar la inflación, pero, además, se encarga de establecer los parámetros para las AFP respecto a los límites de inversión para estas compañías.

Dentro de los organismos del Estado encargados de supervisar y regular el mercado de capitales colombiano encontramos a 3 instituciones, las cuales son:

- El Congreso de la República. - Se encarga de expedir la ley del mercado de valores.

- Ministerio de hacienda y crédito público. - Expide regulaciones que abarcan las leyes generales de la ley del mercado de valores.

- La superintendencia financiera. - Brinda instrucciones sobre el cumplimiento de las normas emitidas en el ministerio de hacienda y crédito público.

Dentro de los antecedentes del mercado bursátil, podemos tomar como referencia el año 1990, ya que en ese periodo llegó el Nuevo Programa Económico que marcó una época de estabilización económica, la cual se tradujo en una mayor capitalización la cual tuvo un periodo de crecimiento anormal pasando a representar de $2.2 \%$ a $20 \%$ en tan solo cinco años, señaló Vinelli (2012).

Un dato importante, es que como señalan Delgado y Humala (1997), a partir de 1990 las acciones inscritas en la BVL mostraron una clara tendencia de crecimiento en su valor fundamental, gracias a la considerable mejora de algunos sectores específicos se amortiguo la salida de varias acciones que cotizaban en la bolsa en aquella época y el incremento de la demanda de acciones generaron que la capitalización bursátil de la BVL aumente.

Además, en el año 1992 se aprobó la reforma del sistema de pensiones lo cual llevaría a una serie de reformas estructurales que Delgado y Humala (1997), señalan como la causa del aumento de la variabilidad de las condiciones de mercado y ya luego hacia 1996 llegaría la apertura comercial y financiera, lo cual hizo que aumente la participación de los inversionistas extranjeros en la BVL. Por ejemplo, la participación 
de estos inversionistas en la Caja de Valores y Liquidaciones (CAVAL) pasó de ser menos de 30 millones de dólares en 1992 a ser 1,500 millones de dólares hacia el 2015.

Posteriormente, fueron apareciendo los fondos mutuos, los fondos de inversión y todo esto llevaría a que se reclasifique el Riesgo País asociado al Perú.

Respecto a la postura de la teoría de la Eficiencia de mercado, Vinelli (2012), menciona que siempre han existido críticas y que estas pruebas buscan detectar patrones de regularidad ya que, si se evidencia la presencia predictibilidad o de anomalías, los inversionistas podrían obtener rendimientos anormales, lo cual llevaría a que se reduzca la credibilidad y por ende la liquidez del mercado bursátil.

Debido a la tendencia que se evidencia en estos indicadores es necesario evaluar las cotizaciones y rendimientos de las empresas más liquidas que cotizan en bolsa y realizar las pruebas para, mediante una modelación, concluir si el mercado peruano ha sido eficiente o no durante el periodo seleccionado. 


\section{MARCO TEÓRICO}

A continuación, se hace referencia de las principales teorías que directa e indirectamente están relacionadas al tema de investigación.

La base teórica está comprendida por la definición de lo que es la eficiencia en el mercado de capitales según Fama y que grados de eficiencia existe y para esto se ha tomado la teoría propuesta por Fama y luego abarcada por French (1980) y Hot (2009) para explicar algunas anomalías que existen en distintas Bolsas de Valores del mundo.

Además, se toma en cuenta el trabajo de Ogege y Mojejkwu (2013), en el que brinda algunas consideraciones a la hora de aplicar la prueba de paseo aleatorio (Random Walk) tomando como ejemplo el mercado bursátil de Nigeria.

Luego de haber definido esto, se pasa a revisar algunos trabajos al respecto en la región, en los que se encuentran documentos como los de Méndez (2009), Morales (2015), Delgado y Humala (1997) y Duarte (2015) para analizar las bolsas de Colombia, Chile y Perú.

También se tomó en cuenta la investigación de los hermanos Zacks, L y Zacks, L (2011), respecto a las anomalías de mercado, en donde todos contrastan la HME de Fama con las finanzas conductuales a fin de ampliar la visión de eficiencia de un mercado bursátil.

\subsection{Hipótesis de Eficiencia de Mercado de Capitales}

A pesar de que existen muchos autores que hablan respecto a la eficiencia de un mercado bursátil, analizada desde un modelo de Juego Justo (Fair Game) hacia inicios de los años 1900, fue recien Fama (1970) quién introdujo esta hipótesis y describió cuales eran las condiciones que este debe cumplir para que pueda ser llamado eficiente. Fama (1970), describe la eficiencia de mercado de la siguiente manera: "security prices at any time "fully reflect" all available information. A market in which prices always "fully reflect" available information is called "efficient." 
La forma débil de eficiencia de mercado. - Bajo esta hipótesis, se afirma que no existe relación entre los precios históricos y los futuros (Fama, 1970), de esta manera se supone que no es posible obtener ganancias de manera sistemática solamente observando el comportamiento de los precios pasados de las acciones.

De manera empírica, si existe eficiencia entonces los precios de las acciones deberían comportarse como un paseo aleatorio (Fama, 1970) para que nadie pueda obtener ganancias utilizando únicamente los precios históricos, es decir:

$$
\mathrm{Pt}=\mu+P t-1+E t
$$

Donde:

- Pt= precio de la acción $x$

- $\mu=$ cambio esperado en el precio

- $\quad$ Et $=$ término de error esperado

La prueba de eficiencia en su forma débil afirma que no existe una relación entre los rendimientos pasados y futuros de los activos.

Los resultados de sus pruebas varían dependiendo del país seleccionado para realizar las estimaciones siendo principalmente los mercados desarrollados los que cumplen estos test.

Algunos de estos estudios como el de Sánchez (2013), muestran predictibilidad en los rendimientos a partir de estimaciones mediante modelos que no deberían ser posibles de acuerdo a la teoría propuesta por Fama, analizando los precios y los rendimientos de algunos índices.

La forma semi-fuerte de eficiencia de mercado. - En esta forma, los precios reflejan toda la información pública disponible y que además tiene coste cero.

Por otro lado, en esta prueba Mackinlay (1997), sostiene que, para probar esta hipótesis, es necesario realizar un estudio de eventos. Además, divulgó unos parámetros con el espíritu de aportar en la comprensión y dinámica de la ejecución de dicha metodología.

Definir el evento de interés e identificar el periodo dentro del cual serán examinados los precios de los instrumentos financieros de las firmas, luego se determina 
los criterios de selección a tener en cuenta para la inclusión de una firma cualquiera en el estudio (para esto se va a utilizar las empresas más liquidas como filtro como ya se mencionó anteriormente).

Al respecto, Ball y Brown (1968), realizan un estudio del anuncio de la utilidad trimestral para ver si las acciones se ajustan a la nueva información disponible. Para esto utilizaron los reportes contables de empresas a fin de estudiar la utilidad de la información que se publica anualmente, llegando a la conclusión que cuando las ganancias son más altas a las predichas, los retornos son sumamente elevados.

La forma fuerte de eficiencia de mercado. - En esta versión de eficiencia, los precios de las acciones reflejan de manera inmediata toda la información pública y privada.

Por otro lado, si esto ocurre, empresas como los fondos mutuos no deberían presentar rendimientos anormales (a pesar de que ellos cuentan con información privilegiada) debido a que, como señala el autor Fama (1970), los precios ajustarán rápidamente la nueva información con lo cual no sería posible que ellos puedan ganarle al mercado de manera sistemática.

Sin embargo, existen costos de transacción que generan que la información tenga un precio por ser privilegiada, esto debido a que los administradores de fondos cuentan con información privilegia que se las otorgan por un costo operativo (Humala y Delgado, 1997) por lo que estas empresas no ven rentable no ven lógico compartir la información con la que cuentan.

\subsection{Supuestos de la hipótesis de mercado eficiente}

Esta hipótesis debe cumplir con ciertos supuestos planteados por (Fama, 1970) y son los siguientes:

- Los precios son libres. - Están determinados por la oferta y demanda entre los agentes superavitarios y los deficitarios y que, además, no interviene ningún organismo o se restringe los precios, esto último parece no estar ocurriendo dado que como señala Chen (2013), existen algunos mercados asiáticos estarían fijando bandas de precio. 
- Existe libre entrada y salida del mercado. - Implica que los agentes son libres de comprar o de vender sus activos en el momento que ellos deseen.

- El mercado provee toda la información relevante sin costo. - Los organismos que administran y regulan este mercado, tratan de que toda la información sea de acceso gratuito para los inversionistas. En esta materia, el avance tecnológico y el internet han significado un gran apoyo para las Bolsas del mundo ya que hoy en día, las compañías publican en sus páginas webs el resultado de su gestión empresarial.

- Los inversionistas hacen uso de toda la información relevante. - Un agente utiliza todo material disponible que encuentre para evaluar el active de su interés.

- El uso que se hace con la información es económicamente correcto. - Esta premisa descansa sobre el hecho de que el agente es un ser racional y sabe cómo utilizar la información para llegar a un precio que se aproxima al valor fundamental.

Sin embargo, también existen algunas limitaciones que surgen a partir de los supuestos para la eficiencia entre las cuales tenemos al número de participantes de mercado, la disponibilidad de la información, costos de transacción, límites a las negociaciones, tamaño de mercado, paradoja valor vs crecimiento.

Entonces, si en un mercado se encuentran estas limitaciones, puede darse el caso de que el mercado bursátil no esté funcionando de manera eficiente.

\subsection{Anomalías de mercado: Efecto fin de semana y Efecto manada}

Una de las ramas de las finanzas que está tomando fuerza con los años pues explica las razones por las cuales los agentes siguen ciertos comportamientos es la de las finanzas conductuales. Sin embargo, antes de que esta rama fuera ganando importancia, French (1980), analiza el efecto fin de semana (The weekend Effect), el cual se define como la probabilidad de obtener rendimientos superiores utilizando un día determinado de la semana para invertir.

Al estimar el modelo a continuación, French (1980), concluye que los rendimientos observados de los días lunes son menores que los otros días, lo cual incumple teoría propuesta por Fama ya que, si un mercado es eficiente, este no debería mostrar estacionalidad en los retornos. 
Utilizando el siguiente modelo y aplicando las pruebas correspondientes:

- $R t=\alpha+\lambda 2 D 2 t+\lambda 3 D 3 t+\lambda 4 D 4 t+\lambda 5 D 5 t$

- Donde:

- $\mathrm{Rt}=$ Retorno de una acción o Índice para un determinado día de la semana

- $\quad \mathrm{D}(\mathrm{it})=$ Variable dummy asociada a un día i $(2,3,4,5)$ de la semana

- $\lambda=$ parámetro que está asociado a partir del día martes

Conclusiones: Al estimar el modelo French concluye que los rendimientos observados de los días lunes son menores que los de otros días. Los resultados obtenidos para algunos índices, como los de las bolsas de Asia, son los que corroboran principalmente este supuesto.

Del mismo modo, si existe cointegración entre mercados (French, 1980), uno puede cambiar de un mercado a otro cuando a uno le está yendo mal y sabe que como el otro está relacionado puede utilizar esto a su favor para predecir algunos movimientos de la bolsa.

Más adelante, Hott (2009), estudia otra anomalía conocida como el efecto manada, la cual se entiende como la posibilidad de que las decisiones tomadas por un grupo de inversionistas tengan un efecto de contagio en otro grupo y que de esta manera ellos se guíen por estas decisiones para invertir dejando de lado el análisis fundamental y contradiciendo el supuesto de racionalidad de Fama (1970).

Al respecto, Hott (2009), define este efecto como la influencia positiva de las decisiones de unos inversores sobre las decisiones de un inversor en particular.

En este marco, existen dos tipos de manada, las cuales son la manada racional y manada irracional.

Manada racional. - Es el conjunto de personas que toman la misma decisión basada en una expectativa racional que es la misma para todos.

Manada irracional. - Es el conjunto de personas que siguen las decisiones del grupo por el hecho de que el precio está tomando tendencias lo cual es irracional.

Interiorizando más en el segundo comportamiento, Hott (2009), argumenta que todo parte de la psicología del agente pues las decisiones que este tome, en cierta parte, 
están influenciadas por los sesgos cognitivos que posea y todo esto genera que, en algún momento dado, el inversor pueda tomar una decisión sin algún sustento fundamental.

Por un lado, Delgado y Humala (1997), mencionan que este comportamiento especulativo de los agentes de corto plazo muchas veces se da porque buscan rentabilizar en la menor cantidad de tiempo, por lo que su manera de pensar y por ende su toma de decisiones es distinta a la de los inversores de largo plazo.

Al respecto, los autores Ouarda, Bouri y Bernard (2013) estudiaron la existencia de este efecto en 10 industrias de la Bolsa Europea (EuroStoxx600) y concluyen que existe este efecto en todos los sectores.

Más adelante, el autor Chen (2013), realizó un estudio entre los años 2000-2009 con varios paises los cuales clasificó como desarrollado, emergentes y de frontera con lo cual concluyó que en la mayoría de ellos se evidenciaba la presencia de la anomalía conocida como el efecto manada.

Es necesario conocer como influyen las decisiones de un grupo de agentes en particular sobre el resto de inversionistas ya que las burbujas del 2001 y 2008 muestran el peso de la especulación, y los rendimientos anormales que obtuvieron algunas empresas y son abarcadas por Shiller (2003), en su libro, el señala que la especulación tuvo un fuerte peso en la caída de la bolsa de Estados Unidos y además que está especulación generó un efecto manada en los inversionistas y que también por las fallas de mercado, los rendimientos que obtuvieron algunos de ellos durante el periodo de la crisis fueron sumamente elevado ya que pudieron detectar estas fallas antes de que el mercado colapse. 


\section{METODOLOGÍA}

Para esto se utilizará un diseño no experimental tomando como periodo de estudio las fechas entre el 01 de enero del 2008 y el 31 de diciembre del 2018, utilizando las cotizaciones diarias del IGBVL25, el COLCAP y además utilizando los rendimientos y la desviación estándar de sección cruzada hallados en el Excel para realizar pruebas de raíz unitaria y varianza en Eviews al método de Mínimos Cuadrados.

Los datos utilizados para detectar la presencia del efecto manada son los índices IGBVL25 y COLCAP, también usados por Chang, Cheng y Khorana (2000), y tomando como medida de referencia la desviación estándar de sección cruzada que va a ser definida a continuación:

$$
\mathrm{CSSD}=\sqrt{\frac{\sum_{i=1}^{N}\left(R_{i, T}-R_{m, T}\right)^{2}}{N-1}}
$$

Donde:

- $\quad \mathrm{CSSD}=$ Desviación estándar de sección cruzada (raíz cuadrada de la diferencia entre los rendimientos reales y esperados)

- $\mathrm{Ri}, \mathrm{T}=$ retorno de la empresa $\mathrm{i}$ en el periodo $\mathrm{t}$

- $\mathrm{Rm}, \mathrm{T}=$ retorno de mercado en el periodo $\mathrm{t}$

Luego, se aplica el siguiente modelo y se utiliza el método de Mínimos Cuadrados:

$$
\operatorname{CSSD}_{t}=\alpha+\beta_{1} D L+\beta_{2} D U+\varepsilon_{t}
$$


Donde:

- $\quad C S S D=$ Desviación estándar de sección cruzada (raíz cuadrada de la diferencia entre los rendimientos reales y esperados)

- $\quad$ DL y DU = Variables Dummy

- $\varepsilon \mathrm{t}=$ término de error

Las pruebas estadísticas a realizar son: Test de Dickey - Fuller (estacionalidad) para demostrar si la serie tiene raíz unitaria y además el Test de varianza. 


\section{ANÁLISIS}

\subsection{Estimación}

Figura 6.1

Mínimos Cuadrados a la CSSD del IGBVL25 con variables DL y

DU, 2008-2018

\begin{tabular}{cccc}
\hline Subtítulo & Coeficiente & Error Estándar & t- estadístico \\
DL & 0.021221 & 0.000702 & 30.20988 \\
DU & 10.021511 & 0.000702 & 30.92299 \\
\hline
\end{tabular}

Nota: La tabla arroja un DW de 1.222957 y un Akaike de -4.454876.

Elaboración propia

Figura 6.2

Mínimos Cuadrados a la CSSD del COLCAP con variables DL y

DU, 2008-2018

\begin{tabular}{cccc}
\hline Subtítulo & Coeficiente & Error Estándar & t- estadístico \\
DL & 0.030763 & 0.000834 & 36.88543 \\
DU & 0.031214 & 0.000834 & 37.43925 \\
\hline
\end{tabular}

Nota: La tabla arroja un DW de 1.041113 y un Akaike de -4.139444.

Elaboración propia

Esto quiere decir que el rendimiento es más significativo hacia los periodos que están dentro del extremo superior de la cola de la distribución. 
Figura 6.3

Test de Raíz Unitaria al IGBVL 25, 2008-2018

\begin{tabular}{cccc}
\hline Subtítulo & Valor crítico & T statistic & Prob \\
DF Aumentado & & -10.01431 & 0.000 \\
Fila 1 & $1 \%$ & -3.432537 & \\
Fila 2 & $5 \%$ & -2.862392 & \\
Fila 3 & $10 \%$ & -2.567268 & \\
\hline
\end{tabular}

Nota: La tabla muestra la prueba de raíz unitaria y el estadístico hallado al 1\%, 5\%, 10\%.

Elaboración propia

Figura 6.4

Test de Raíz Unitaria a COLCAP, 2008-2018

\begin{tabular}{cccc}
\hline Subtítulo & Valor crítico & T statistic & Prob \\
DF Aumentado & & -14.46358 & 0.000 \\
Fila 1 & $1 \%$ & -3.432601 & \\
Fila 2 & $5 \%$ & -2.862420 & \\
Fila 3 & $10 \%$ & -2.567283 & \\
\hline
\end{tabular}

Nota: La tabla muestra la prueba de raíz unitaria y el estadístico hallado al 1\%, 5\%, 10\%.

Elaboración propia

Con lo cual se rechaza la hipótesis nula al 0.05 de que la CSSD sigue un comportamiento aleatorio. 
Figura 6.5

Test de Varianza a IGBVL 25

\begin{tabular}{|c|c|c|c|}
\hline Subtítulo & Periodo & Var ratio & Value \\
\hline $\begin{array}{l}\text { Joint Tests: Max z } \\
\text { (al periodo 2) }\end{array}$ & & & 10.51498 \\
\hline Fila 1 & 2 & 0.473304 & \\
\hline Fila 2 & 4 & 0.263556 & \\
\hline Fila 3 & 8 & 0.149719 & \\
\hline Fila 4 & 16 & 0.072490 & \\
\hline
\end{tabular}

Nota: La tabla muestra la prueba de varianza con intervalos de 2, 4, 8 y 16 periodos.

Elaboración propia

Figura 6.6

Test de Varianza a COLCAP

\begin{tabular}{cccc}
\hline $\begin{array}{c}\text { Subtítulo } \\
\text { Joint Tests: Max z } \\
(\text { al periodo 2) }\end{array}$ & Periodo & Var ratio & Value \\
Fila 1 & 2 & 0.986027 \\
Fila 2 & 4 & 0.356589 & \\
Fila 3 & 8 & 0.196362 & \\
Fila 4 & 16 & 0.097679 & \\
\hline
\end{tabular}

Nota: La tabla muestra la prueba de varianza con intervalos de 2, 4, 8 y 16 periodos.

Elaboración propia

La prueba Individual muestra la varianza a través del tiempo y como se puede apreciar en la Tabla 5, el ratio de varianza va descendiendo conforme pasan los periodos, lo que quiere decir que la CSSD se estabiliza cuando pasan muchos rezagos. 


\subsection{Interpretación Económica}

Luego de obtener los resultados y compararlos con los hallados por Duarte, Garcés y Sierra (2015), y utilizando la metodología de Chang, Cheng y Khorana (2000) se detectó la presencia de un efecto manda en el IGBVL25 y COLCAP principalmente durante los años posteriores a la crisis financiera del 2008, es decir, que la ambas atrajeron mucho capital extranjero dado que estos inversionistas buscaron rentabilizar en mercados como estos y así generando esta anomalía.

Tal y como señalan los autores Duarte, Garcés y Sierra (2015), este efecto ocurre frecuentemente cuando los mercados bursátiles latinoamericanos están al alza (Perú y Colombia), lo cual demuestra que los inversionistas responden de manera rápida ante las noticias de este tipo, así como también lo planteaba el autor Chen (2013).

Por último, estos autores utilizan dos metodologías distintas para hallar la presencia de este efecto, encontrando en una metodología general que lo rechazaba. Sin embargo, luego utiliza la metodología de Chang, Cheng y Khorana (2000) la cual es la que ha sido replicada en este trabajo, dado que como señala el autor, esta es más precisa para detectar la presencia de este efecto y que es mejor que el otro modelo que planteó anteriormente.

Sin embargo, para el efecto manada se encuentran resultados ambiguos ya que tras utilizar una de las metodologías planteadas por el autor (la cual señala que es la más precisa para detectar este efecto) se encontró la evidencia de un efecto manada pero cuando utiliza la otra metodología obtiene que no hay evidencia de un efecto manada.

Otro punto a considerar es que como señala Duarte, Garcés y Sierra (2015), este modelo es lineal lo cual no es una condición de los supuestos de Fama (1970).

En conclusión, tras realizar las pruebas de raíz unitaria y de varianza, se comprueba que los índices de la BVL y la BVC presentan estacionalidad y predictibilidad lo cual permite a inversores obtener rendimientos anormales más probablemente en épocas de crisis ya que estos se adelantan al mercado y mueven sus capitales a otras bolsas más probablemente de mercados emergentes como el nuestro. 


\section{CONCLUSIONES}

- Para el efecto manada se encontró la evidencia de este en el corto plazo, que va despareciendo a través del tiempo, esto debido a que, a partir de la crisis financiera del 2008, hubo una atracción por parte de los inversionistas hacia las bolsas de mercados emergentes (entre las cuales se encuentra la BVL y la BVC), lo cual tuvo un fuerte impacto en el incremento de ambas.

- Las crisis financieras evidencian que los agentes económicos dejan de lado la parte racional (Chen, 2013) y se comportan en manadas (positivas o negativas) que muestran que el mercado bursátil no es eficiente y a pesar de que al inicio beneficia a un grupo específico de inversionistas, el resto del grupo (manada) sigue las decisiones que conllevan con rendimientos a favor en menor escala de todos. 


\section{RECOMENDACIONES}

- Se recomienza analizar qué factores de la política económica de un país toman mayor relevancia dentro de los inversionistas y que puedan desencadenar una crisis financiera y otro efecto manada.

- Se recomienda aumentar las investigaciones de las finanzas conductuales para evidenciar los casos en los que el agente económico actúa de manera irracional dado que la hipótesis de los mercados eficientes tiene como supuesto de que el agente económico es siempre racional.

- Se recomienda desagregar los sectores que conforman las bolsas y poder realizar un estudio en un periodo de tiempo más grande que permita evaluar más de 1 crisis para corroborar que es una tendencia que se repite y en cuales es donde los inversionistas se refugian luego de iniciadas las crisis. 


\section{REFERENCIAS}

Banco Central de Reserva del Perú. (2016). Reporte de Estabilidad Financiera. Lima: Autor.

Banco Central de Reserva del Perú. (2018). Reporte de Estabilidad Financiera. Lima: Autor.

Banco de la República Bogotá. (2018). Reporte de Estabilidad Financiera. Bogotá: Autor.

Bolsa de Valores de Lima. (31 de 12 de 2018). Información estadística 2013 -2018.

Recuperado de Bolsa de Valores de Lima web site: https://www.bvl.com.pe/estadist/dat_infostat.pdf

Chang, E., Cheng, J., y Khorana, A. (2000). An examination of herd behavior in equity markets: An international perspective. Journal of Banking y Finance, 24(10), 1651-1679. doi:10.1016/S0378-4266(99)00096-5

Chen, T. (2013). Do investors herd in global stock markets? Journal of Behavioural Finance, 14(3), 230-239. doi:10.1080/15427560.2013.819804

Choy, M., y Chang, G. (2014). Medidas macroprudenciales aplicadas en el Perú (DT. $N^{\circ}$ 2014-007). Lima: Banco Central de Reserva del Perú. Recuperado de http://www.bcrp.gob.pe/docs/Publicaciones/Documentos-deTrabajo/2014/documento-de-trabajo-07-2014.pdf

De Almeida, R., Costa, H., y Da Costa, N. (2012). Herd behavior in Latin American stock markets. Latin American Business Review, 13(2), 81-102. doi:10.1080/10978526.2012.700271

Delgado, L., y Humala, A. (1997). Banco Central de Reserva del Perú. Recuperado de Banco Central de Reserva del Perú website: http://www.bcrp.gob.pe/docs/Publicaciones/Documentos-deTrabajo/1997/Documento-Trabajo-04-1997.pdf 
Duarte, J., Garcés, L., y Sierra, K. (2015). Efecto manada en sectores económicos de las bolsas latinoamericanas: una visión pre y poscrisis subprime. Universidad Nacional Autónoma de México, 61(2), 298-323. doi:10.1016/j.cya.2015.12.002

Fama, E. (1970). Efficient Capital Markets: A Review of Theory and Empirical Work. The Journal of Finance, 25(2), 383-417. doi:10.2307/2325486

French, K. R. (1980). Stock Returns and the Weekend Effect. Journal of Financial Economics, 8(1), 55-69. doi:10.1016/0304-405X(80)90021-5

Gebka, B., y Wohar, M. (2013). International Herding: Does it differ across sectors? Journal of International Financial Markets, Institutions and Money, 23, 55-84. doi:10.1016/j.intfin.2012.09.003

Henker, J., Henker, T., y Mitsios, A. (2006). Do investors herd intraday in Australian equities? International Journal of Managerial Finance, 2(3), 196-219. doi:10.1108/17439130610676475

Hott, C. (2009). Herding Behavior in assets markets. Journal of Financial Stability, 5(1), 35-56. doi:10.1016/j.jfs.2008.01.004

Khan, W., y Vieito, J. (2012). Stock exchange mergers and weak form of market efficiency: the case of Euronext Lisbon. International Review of Economics \& Finance, 22(1), 173-189. doi:10.1016/j.iref.2011.09.005

Lao, P., y Singh, H. (2011). Herding behaviour in the Chinese and Indian stock markets. Journal of Asian Economics, 22(6), 495-506. doi:10.1016/j.asieco.2011.08.001

Liu, S. (2013). An empirical analysis of herd behavior in the Singapore stock market (degree of Master). Saint Mary’s University. Recuperado de https://pdfs.semanticscholar.org

Mackinlay, C. (1997). Event Studies in Economics And Pennsylvania. Journal of Economic Literature, 35, 13-39. Recuperado de https://pdfs.semanticscholar.org/3c84/c0f2c504dd84e7fee6f124c2ea5f8afb706e. pdf

Mendez, A. (2009). Revisión a la Eficiencia del mercado de capitales colombiano. Medellín: Universidad Nacional de Colombia. Recuperado de http://www.scielo.org.co/pdf/seec/v17n35/v17n35a2.pdf 
Morales, J., Ruíz, E., y Gutiérrez, B. (2015). Hipótesis de mercados eficientes en el mercado accionario colombiano a través del estudio de eventos. Antioquia: Congreso de contaduría, administración e informática. Recuperado de http://congreso.investiga.fca.unam.mx

Ouarda, M., Bouri, A., y Bernard, O. (2013). Herding Behaviour under markets condition: Empirical evidence on the European financial markets. International Journal of Economics and Financial Issues, 3(1), 214-228. Recuperado de https://www.econjournals.com

Rodríguez, W. (2012). Day Of The Week Effect In Latin American Stock Markets. Revista de Análisis Económico, 27(1), 71-89. doi:10.4067/S071888702012000100004.

Sánchez, J. W. (28 de Octubre de 2013). Banco Central de Reserva del Perú. Recuperado de Banco Central de Reserva del Perú Website: http://www.bcrp.gob.pe/docs/Proyeccion-Institucional/Encuentro-deEconomistas/2013/ee-2013-sanchez.pdf

Shiller, R. (2003). From Efficients Markets Theory to Behavioral Finance. The Journal of Economics Perspectives, 17(1), 83-104. Recuperado de http://www.its.caltech.edu/ camerer/Ec101/EffMktsThryBehFin_Shiller_Win03 .pdf

Shynkevich, A. (2012). Short- term predictability of equity returns along two style dimensions. Journal of Empirical Finance, 19(5), 675-685. doi:10.1016/j.jempfin.2012.07.003

Vinelli, M. A. (27 de 06 de 2012). Banco Central de Reserva del Perú. Recuperado de Banco Central de Reserva del Perú web site: http://www.bcrp.gob.pe/docs/Proyeccion-Institucional/Encuentro-deEconomistas/XXV-EE-2007/XXV-EE-2007-S06-Sanchez-Vinelli.pdf 


\section{BIBLIOGRAFÍA}

Bollerslev, T. (1986). Generalized Autoregressive Conditional Heteroskedasticity. Journal of Econometrics, 31(3), 307-327. doi:10.1016/0304-4076(86)90063-1

Ogege, S., y Mojekwu, J. (2013). Econometric Investigation of the Random Walk Hypothesis in the Nigerian Stock Markets. Journal of Emerging Issues in Economics, Finance and Banking (JEIEFB), 1(5), 381-400. Recuperado de https://pdfs.semanticscholar.org

Straßburg, J., Gonzáles, C., y Alexandrov, V. (2012). Parallel genetic algorithms for stock market trading rules. Procedia Computer Science, 9, 1306-1313. doi:10.1016/j.procs.2012.04.143

Zacks, L., y Zacks, L. (2011). The handbook of Equity Market Anomalies. New Jersey: John Wiley \& Sons, Inc. doi:10.1177/2319510X13482014 
ANEXOS 


\section{Anexo 1: Matriz de Operacionalización para Efecto Manada}

\begin{tabular}{|c|c|c|c|c|}
\hline $\begin{array}{c}\text { Variables } \\
\text { seleccionadas }\end{array}$ & Indicador & Fuente & Variable & $\begin{array}{c}\text { Diseño no } \\
\text { experimental }\end{array}$ \\
\hline $\begin{array}{l}\text { Desviación } \\
\text { Estándar de } \\
\text { sección cruzada }\end{array}$ & $\begin{array}{l}\text { Raíz cuadrada del } \\
\text { cociente de los } \\
\text { rendimientos } \\
\text { reales y } \\
\text { esperados y la } \\
\text { covarianza }\end{array}$ & www.bloomberg.com & Explicativa & $\begin{array}{l}\text { Corte trasversal. } \\
\text { Datos diarios y } \\
\text { mensuales } 2008- \\
2018 .\end{array}$ \\
\hline DL, DU & $\begin{array}{c}\text { Valores entre } 0 \mathrm{y} \\
1\end{array}$ & Elaboración propia & Dummy & $\begin{array}{c}\text { Datos diarios y } \\
\text { mensuales } 2008- \\
2018\end{array}$ \\
\hline
\end{tabular}

Elaboración propia 